\title{
Advanced Heterojunction Structure of Polymer Photovoltaic Cell Generating High Photocurrent with Internal Quantum Efficiency Approaching 100\%
}

\author{
Hui Joon Park, Jae Yong Lee, Taehwa Lee, and L. Jay Guo*
}

Polymer photovoltaic (PV) cells are promising green energy sources due to the potential for low-cost production of flexible solar cells over a large area with acceptable efficiencies. ${ }^{[1-12]}$ Among various photoactive layer structures for polymer PV cells, bulk heterojunction (BHJ) structure has been exploited as one of the most successful structures giving the highest efficiencies by the formation of interpenetrating nanoscale networks of electron-donor (D) and electron-acceptor (A). ${ }^{[2-7]}$ Therefore, significant efforts have been made to optimize and understand those structures. However, the random nature of the phase separated D/A domains, based on the simple blending of the two components, limits further optimization of the structures as well as thorough understandings of their physical properties.

To construct the efficient photoactive layers, they should be sufficiently thick to maximize the light absorption and photogenerated excitons; and these excitons should be dissociated efficiently to produce free charge carriers. Furthermore, charge recombination should be minimized during the charge transport to the electrodes. Thicker organic PV layers (hundreds of nanometers thickness) can not only ensure the light absorption but can also have better film thickness control from manufacturing point of view. But as the thickness increases to this range, the low carrier mobility of organic semiconductors and increased probability of forming isolated donor and acceptor islands within the $\mathrm{BHJ}$ film inevitably induce poor charge transportation with high recombination, which results in low internal quantum efficiency (IQE) and low fill factor. As a result, the power conversion efficiency (PCE) does not improve with increasing layer thickness. Therefore, there is a need to

Dr. H. J. Park, ${ }^{[+]}$Prof. L. J. Guo

Macromolecular Science and Engineering

The University of Michigan

Ann Arbor, Michigan 48109, USA

E-mail: guo@umich.edu

J. Y. Lee, Prof. L. J. Guo

Electrical Engineering and Computer Science

The University of Michigan

Ann Arbor, Michigan 48109, USA

T. Lee, Prof. L. J. Guo

Mechanical Engineering

The University of Michigan

Ann Arbor, Michigan 48109, USA

[+] Present address: Portland Technology Development, Logic Technology Development,

Intel Corporation, Hillsboro, Oregon 97124, USA

DOI: 10.1002/aenm.201300245 search for structures superior to the simple blended-BHJ structures to realize thicker and more efficient PVs. For this purpose, we introduce a new type of heterojunction structure and the associated fabrication technique, which produces improved charge transport and higher efficiency even in semiconductor films of hundreds of nanometer thickness.

The bilayer structure of electron-donor and electronacceptor is a simple structure that can help easy optimization of devices and better understanding of their physical properties. However, it is known to be an inefficient architecture for organic PV (OPV) cells, because of the limited photo-carriers generation due to rather short exciton diffusion length and the limited interfacial areas between the donor and acceptor domains responsible for the charge separation. Therefore their device performances cannot compare with those of BHJ-based PV cells. Recent works have reported that by using solution casting to increase the intermixing of donor and acceptor layers, the bilayer approach could mimic the BHJ structure; however, the structure could only utilize tens of nanometers thin donor layer to allow efficient interdiffusion of the acceptor, which was not sufficient to fully absorb the light. Thus, their performances could not exceed those of conventional BHJ-based devices. ${ }^{[13,14]}$

In contrast, the polymer PV cell introduced here, which is fabricated by a bilayer-like approach and composed of the optimized heterojunction structure, can approach almost 100\% IQE at certain wavelength range even with over $300 \mathrm{~nm}$ thick electron-donor layer, producing higher photocurrent than conventional $\mathrm{BHJ} \mathrm{PV}$ cells can achieve. The key innovation of such an approach is that by using a sufficiently thick donor layer and a fabrication process that allows efficient interdiffusion of acceptor molecules into the thick donor phase, wellorganized donor and acceptor nano-domains can be formed. The morphology of the film, characterized by grazing incidence wide angle X-ray scattering (GIWAXS), atomic force microscopy (AFM) and scanning electron microscope (SEM), shows superior characteristics to the conventional BHJ structures, which benefits the charge transport and minimizes the bimolecular recombination of the charge-separated electrons and holes. The principle of our approach is demonstrated by a model system using poly(3-hexylthiophene) (P3HT) and $[6,6]$-phenyl- $\mathrm{C}_{61}$-butyric acid methylester (PCBM), which are the most widely studied commercially available materials. The P3HT:PCBM polymer PV cells produced by our new process show longer bimolecular life-time of photo-carriers and higher internal quantum efficiency (IQE), indicating that the nanostructures in photoactive layer are well-optimized. Therefore resultant short circuit currents of these devices are over $50 \%$ higher than those of the thermally annealed $\mathrm{BHJ}$ polymer PV 
(a)

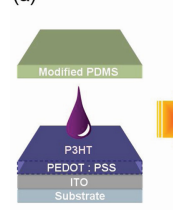

(b)

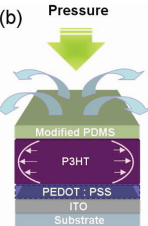

(c)

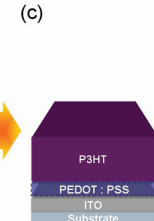

(d)

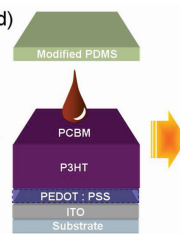

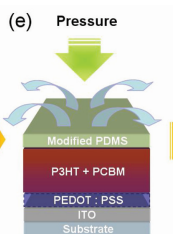

(f)

www.MaterialsViews.com

Figure 1. Schematic of the 2-steps ESSENCIAL process for the advanced heterojunction structure of polymer PV cell: (a) Applying P3HT solution in chlorobenzene; (b,c) P3HT layer formation during solvent evaporation under pressure; (d) Applying PCBM solution in dichloromethane; (e) Active layer formation during solvent evaporation under pressure; (f) Isolated island-type electrode deposition on top of photoactive layer after removing the gas-permeable stamp. Any post annealing process such as thermal treatment is not needed.

cells. Structures fabricated by different methods are further compared by the measurements of external quantum efficiency (EQE), absorbance spectra, dynamic secondary ion mass spectroscopy (DSIMS), and carrier extraction by linearly increasing voltage (CELIV) method. This approach and the new process suggest an alternative and promising method toward high efficiency polymer PV cells.

The overall processing is composed of two steps, as depicted in Figure 1. Unlike the simple spin-casting process used in the previous works to produce intermixed bilayer structures, ${ }^{[13,14]}$ we employ an alternative fabrication process recently developed in our lab. We have demonstrated that BHJ blend could be optimized to have improved and uniform distribution in the vertical direction when it was casted using a gas-permeable cover layer, the so called ESSENCIAL process, which stands for evaporation of solvent through surface encapsulation and induced alignment of polymer chains by applied pressure. Detailed description of the method and $\mathrm{BHJ}$ characterizations can be found elsewhere. ${ }^{[7]}$ In this work, we report a bilayerlike device where the donor and acceptor layers are applied in separate steps by using the ESSENCIAL process, which show significantly improved performance for relatively thick P3HT:PCBM OPV devices. To fabricate such a structure, firstly, the donor P3HT layer is formed using the ESSENCIAL process. In this process, the P3HT solution in chlorobenzene is placed on a transparent anode substrate coated with hole transporting layer poly(3,4ethylenedioxythiophene):poly(styrenesulfo nate) (PEDOT:PSS), and capped with a gas-permeable silicone film, to which a slight pressure is applied. The induced shear flow helps the alignment of the polymer chains while the solvent evaporates through the silicone film. The solidified P3HT layer (about $350 \mathrm{~nm}$ ) remains on the substrate after removal of the silicone film. The thickness of the film can be controlled by changing the concentration of the solution and the applied pressure. ${ }^{[7]}$ A shear stress applied to the polymer solution which helps the organization of the polymer - across the entire depth between the two plates is much more effective than that between a plate and an air surface (as, for example, in the spin-casting process), which decreases continuously from the plate to zero at the air interface. ${ }^{[15]}$ This means that by using ESSENCIAL process, well-organized P3HT polymer domains can be well-maintained even at the top surface, which is clearly evident from the SEM and AFM characterizations (Figure 2a, c and e). The SEM image in Figure 2a clearly shows continuous P3HT nanowires with majority nanofibrils of $\sim 20 \mathrm{~nm}$ in width and exceeding hundreds of $\mathrm{nm}$ in length. These features are also clearly shown in AFM topographical and phase images (Figure 2c and e). This is in sharp contrast to the P3HT film morphology formed by spin-casting, or even in the thermally annealed film, where the organized P3HT nanodomains are hardly discernible at the surface of the film (Figure $2 b, d$ and $f$ ). We believe such change in film morphology can greatly facilitate the charge transport and minimize bimolecular recombination. This is because, in the final completed device with cathode on top, the photo-generated holes near the cathode and throughout the film can travel very efficiently through the fibril P3HT nanocrystals to reach the bottom anode. In other words, the well-formed P3HT crystals form efficient "highways" for the hole carriers in such a thick PV cell. In comparison, in the conventional $\mathrm{BHJ}$ devices, isolated $\mathrm{P} 3 \mathrm{HT}$ islands are inevitable formed, which not only restrain the charge transport to the electrode, but also increase the probability of charge recombination at such isolated islands. Therefore, we anticipate higher quantum efficiency in the new device structure, and increased photo-current level.

The ordering of P3HT polymer chains is further investigated by GIWAXS. In this experiment, the samples were measured at $0.3^{\circ}$ over the critical angle to analyze the organization of polymer chains in the bulk. ${ }^{[14]}$ As shown in GIWAXS patterns (Figure $2 \mathrm{~g}$ and Figure $2 \mathrm{~h}$ ), the strong (h00) signals, which are designated by first order (100), second order (200) and third order (300) diffraction, were detected in the out-of-plane scattering (Figure 2g), and (010) in-plane scattering signal was also shown (Figure $2 \mathrm{~h}$ ), which mean that most of P3HT polymer chains have the edge-on morphology. ${ }^{[16]}$ The P3HT film formed by the ESSENCIAL process shows stronger intensity of both (h00) and (010) signals, which means better organization of P3HT crystal nanodomains. The thicknesses of P3HT layers formed by both ESSENCIAL and spin-casting are the same (about $350 \mathrm{~nm}$ ) for comparison.

On top of the P3HT film with well-organized nanodomains, a PCBM acceptor phase is formed using a second step ESSENCIAL process. We will show that this second ESSENCIAL step helps to maximize the interdiffusion of PCBM into P3HT donor layer. The sequential casting of the bilayer-like structure is made possible by using a solvent for PCBM that is orthogonal to the P3HT donor, such as dichloromethane. In order to characterize the amount of PCBM that penetrated into P3HT domains through the photoactive layer, DSIMS was utilized to determine the relative concentration of P3HT and PCBM across the film thickness (Figure 2i and Figure 2j). For this measurement, deuterated PCBM having ${ }^{2} \mathrm{H}$ atoms that provided the strong signal 

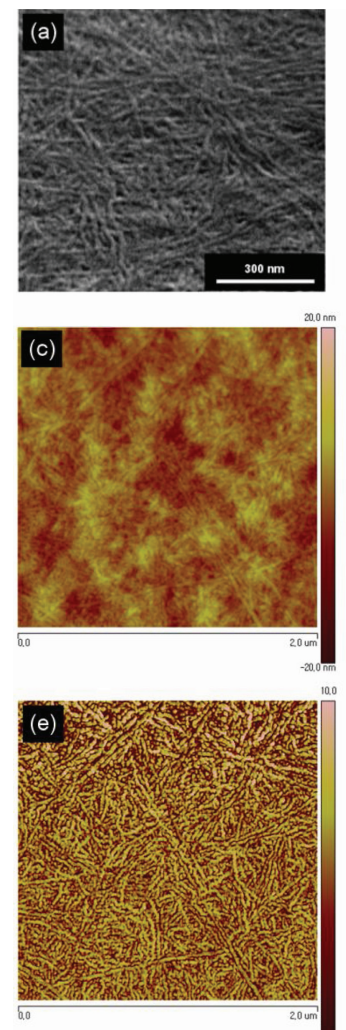
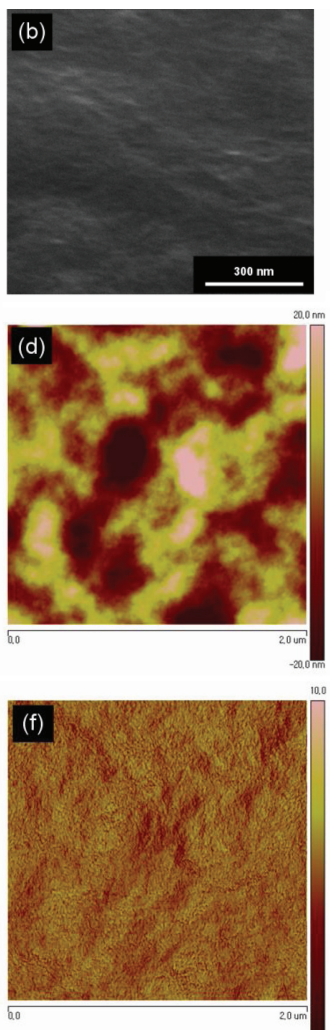
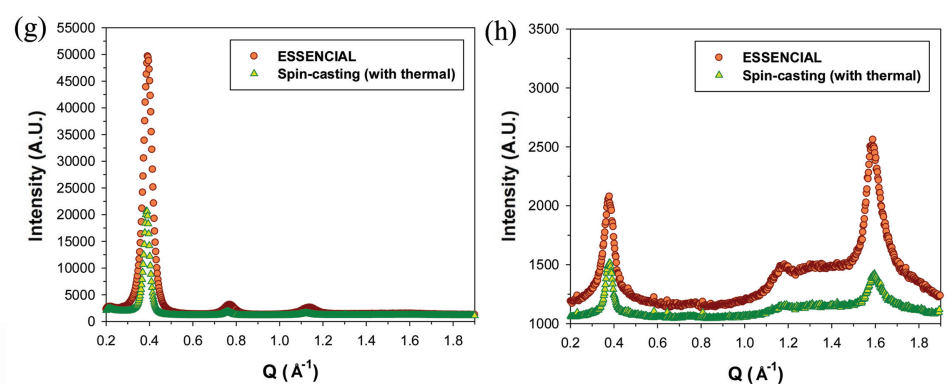

(i)
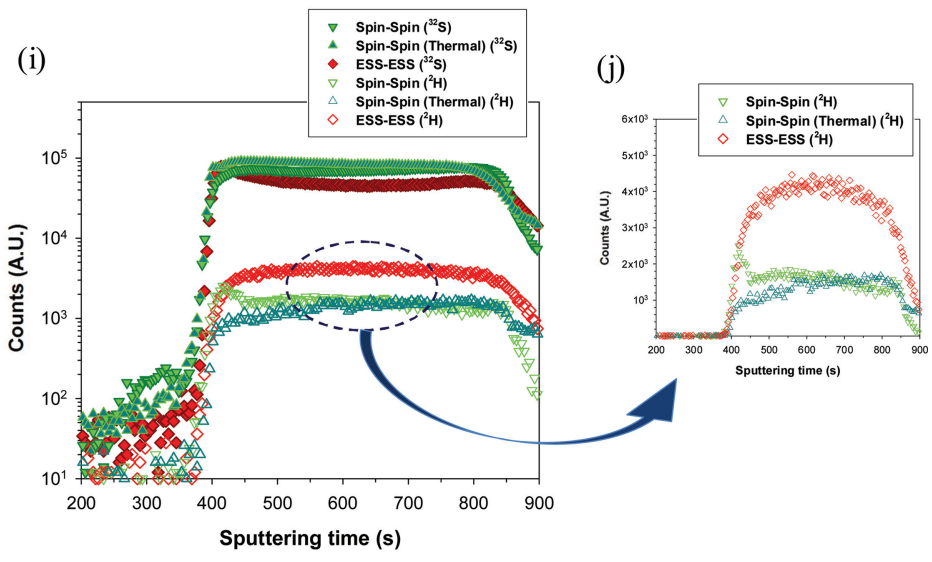

Figure 2. Comparison of P3HT nanodomains fabricated by ESSENCIAL (a, c, e, g) process and those by spin-casting followed by thermal annealing (b, d, f, h) by SEM, AFM, and DSIMS characterization: (a), (b) SEM images; (c), (d) AFM height images; (e), (f) AFM phase images; (g), (h) GIWAXS signals (g), diffracted X-ray intensity in the out-of-plane direction; (h), diffracted X-ray intensity in the in-plane direction). The thicknesses of P3HT layers formed by both ESSENCIAL and spin-casting are the same $(350 \mathrm{~nm})$ for comparison. DSIMS results of photoactive layers composed of P3HT and deuterated PCBM (i, j). Inversed triangle, triangle, diamond represent the films made by spin-casting/spin-casting, spin-casting/spin-casting followed by thermal annealing, and ESSENCIAL/ESSENCIAL. Filled symbols and open symbols represent ${ }^{32} \mathrm{~S}$ for P3HT and ${ }^{2} \mathrm{H}$ for deuterated PCBM, respectively: (i) log scale; (j) normal scale.

to DSIMS measurement was used to form the bilayer-like photoactive layer. The composition of P3HT in the photoactive layer was monitored by the ${ }^{32} \mathrm{~S}$ signal. ${ }^{[14,17]}$ As a comparison, control samples where the PCBM was applied by conventional spin-coating were included in this study. The thicknesses of P3HT layers in all cases are the same. Similar to the recent reports, we found that PCBM can diffuse throughout the P3HT layer during spin-casting (inverse triangle in Figure. Figure $2 \mathrm{i}$ and Figure 2j). ${ }^{[17]}$ Even though further thermal annealing after spin-casting of PCBM (triangle symbol) is beneficial to the migration of PCBM into the P3HT layer, the amount of PCBM that can penetrate into the P3HT layer is limited. In contrast, the ESSENCIAL process that can provide much longer solvent dwelling time during the coating step significantly increases the amount of PCBM (about three times) diffused into the P3HT layer, even without any post annealing process (diamond symbol). Based on a recent report that PCBM molecules diffuse through the amorphous phase of P3HT polymers during annealing process without much impact to the crystal domains of P3HT, ${ }^{[14,17]}$ we anticipate that during the second ESSENCIAL process large amount of PCBM molecules diffuse through the amorphous region of the P3HT around the crystallized P3HT nanowire domains. This process helps to form crystalized
PCBM domains surrounding the P3HT nanowires providing a quite ideal D/A domain morphology for efficient exciton dissociation. This will be discussed again with device performances. In $\mathrm{BHJ}$ blends, the isolated nanodomains are easily generated due to their random nature, and this is particularly evident as the thickness of blends is increased. As a result, typically the BHJ PV cells show the tendency of reduced fill factor with the increased blend thickness, ${ }^{[6]}$ however, using our new process, the donor phase is firstly formed with well-organized donor nanowires and the acceptor molecules spontaneously form continuous pathways during their diffusion into the donor phase, thereby approaching a quasi bi-continuous phase between the donor and acceptor domains. Such a bi-continuous phase distributed in a hundreds of nanometers thick film provides an efficient, heterojunction-like structure, which is advantageous for efficient light absorption and charge separation and transport. Consequently, the improved crystallinity of the donor phase, which comes from the first ESSENCIAL step, and the efficient heterojunction nanodomains as a result of the second ESSENCIAL step, give superior morphology even in hundreds of nanometers photoactive layer. We expect improved charge carrier mobility in such a bi-continuous phase structure, which can give rise to reduced bimolecular recombination process 

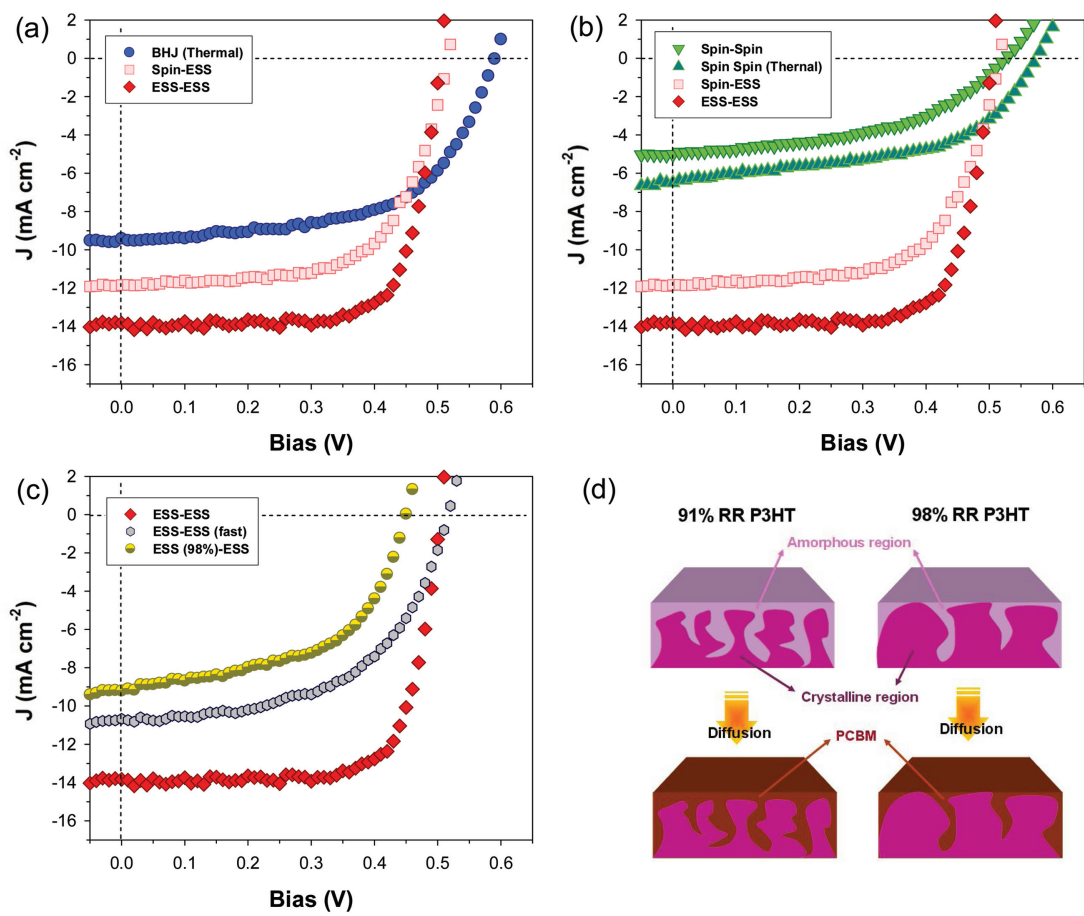

(d)

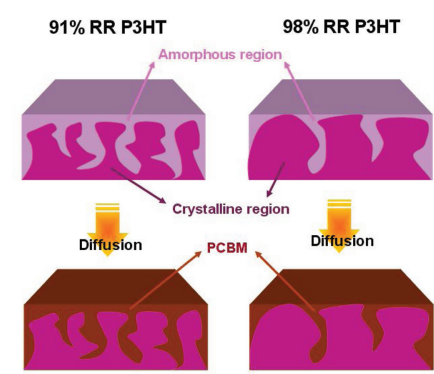

(e)

\begin{tabular}{|c|c|c|c|c|}
\hline Method & $J_{\mathrm{sc}}\left[\mathrm{mA} \mathrm{cm} \mathrm{cm}^{-2}\right]$ & $V_{o c}[\mathrm{~V}]$ & $\mathrm{FF}[\%]$ & PCE [\%] \\
\hline BHJ (Thermal annealed) & $9.38 \pm 0.44$ & $0.59 \pm 0.00$ & $58.96 \pm 1.20$ & $3.27 \pm 0.17$ \\
\hline Spin-Spin & $5.32 \pm 0.54$ & $0.52 \pm 0.02$ & $47.87 \pm 2.06$ & $1.34 \pm 0.17$ \\
\hline Spin-Spin (Thermal annealed) & $6.57 \pm 0.87$ & $0.58 \pm 0.00$ & $47.28 \pm 3.62$ & $1.80 \pm 0.31$ \\
\hline Spin-ESS & $11.84 \pm 0.70$ & $0.54 \pm 0.03$ & $60.98 \pm 3.89$ & $3.91 \pm 0.30$ \\
\hline ESS-ESS & $13.83 \pm 0.52$ & $0.51 \pm 0.01$ & $66.98 \pm 5.05$ & $4.71 \pm 0.36$ \\
\hline ESS-ESS (Fast evaporation) & $10.91 \pm 1.02$ & $0.53 \pm 0.01$ & $53.67 \pm 7.45$ & $3.07 \pm 0.42$ \\
\hline ESS (98\% rr)-ESS & $8.70 \pm 0.74$ & $0.46 \pm 0.01$ & $44.06 \pm 0.31$ & $1.97 \pm 0.41$ \\
\hline \multicolumn{2}{|l|}{ Method } & \multicolumn{3}{|c|}{ Carrier mobility $\left[10^{-4} \mathrm{~cm}^{2} \mathrm{~V}^{-1} \mathrm{~s}^{-1}\right]$} \\
\hline \multicolumn{2}{|c|}{ BHJ (Thermal annealed) } & \multicolumn{3}{|c|}{0.35} \\
\hline \multicolumn{2}{|l|}{ Spin-ESS } & \multicolumn{3}{|c|}{0.61} \\
\hline \multicolumn{2}{|l|}{ ESS-ESS } & \multicolumn{3}{|c|}{1.58} \\
\hline
\end{tabular}

Figure 3. (a)-(c) J-V plots of PV cell devices according to the different fabrication processes. Blue color circle, square, diamond, inverse triangle, triangle, grey color circle, and half yellow color circle represent BHJ (thermally annealed), Spin-ESS, ESS-ESS, Spin-Spin, Spin-Spin (thermally annealed), ESS-ESS (fast evaporation of solvent) and ESS-ESS (98\% rr-P3HT). All data were measured at AM $1.5 \mathrm{G}$ with an intensity of $100 \mathrm{~mW} \mathrm{~cm}{ }^{-2}$. (d) Schematic of diffusion process of $\mathrm{PCBM}$ into $\mathrm{P} 3 \mathrm{HT}$ layers having different regioregularity during second ESSENCIAL process. (e) Summary of device performances according to the different processing methods. (f) Calculated carrier mobilities by photo-CELIV depending on the different processing methods.
www.MaterialsViews.com

in a thick OPV device leading to increased photocurrent. Certainly the speculation of a quasi bi-continuous phase will need to be substantiated with further structural characterization, e.g. TEM tomography, but we will provide transport data later from photoCELIV results to verify the improved charge transport and reduced recombination.

The polymer PV devices fabricated by different processing techniques are characterized and their $\mathrm{J}-\mathrm{V}$ curves are displayed in Figure 3, their performances summarized in Figure 3e. To compare with conventional $\mathrm{BHJ}$ devices, thermally annealed $\mathrm{BHJ}$ devices with $320 \mathrm{~nm}$ thick photoactive layer were chosen as they showed the highest performances (Figure S1a and S1b), which had PCEs around $3.3 \%$ with $9.38 \pm 0.44 \mathrm{~mA} \mathrm{~cm}^{-2}$ short circuit current $\left(U_{\text {sc }}\right)$ and $58.96 \pm 1.20 \%$ fill factor (FF). Compared with these PV cells, the devices, which are fabricated by two steps consecutive ESSENCIAL processes (denoted as ESS-ESS), having the optimized morphology show high $J_{\mathrm{sc}}\left(13.83 \pm 0.52 \mathrm{~mA} \mathrm{~cm}{ }^{-2}\right)$ and FF $(66.98 \pm 5.05 \%)$ for the P3HT:PCBM system. Even the devices, of which only PCBM phase is formed by ESSENCIAL process on the spin-casted P3HT domains (denoted as Spin-ESS), also show high $J_{\mathrm{sc}}$ (11.84 $\pm 0.70 \mathrm{~mA} \mathrm{~cm}{ }^{-2}$ ) (Figure 3a). As shown in Figure 3b, Spin-Spin PV cells, fabricated by spin-casting the PCBM layer on top of hundreds of nanometers thick P3HT layer, cannot produce optimal heterojunction structure, even followed with thermal annealing. This is because this approach cannot impart sufficient amount of PCBM into P3HT domains by interdiffusion in this thickness range. In contrast, if the PCBM phase is formed by the ESSENCIAL step, the increased solvent dwelling time during this process significantly improves acceptor molecule diffusion into the donor phase, which results in improved Spin-ESS device performances. Furthermore, the ESS-ESS devices provide additional benefit by having well-organized P3HT nanodomains from the first ESSENCIAL step, leading to further improvement of $J_{\mathrm{sc}}$ and FF. To verify that the solvent evaporation time is a crucial parameter in this process, we fabricated PV cells by forming PCBM phase using gas-permeable stamp that had faster solvent evaporation speed (and comparable to spin-casting time). The device performances were dramatically degraded as shown in Figure 3c. This illustrates that the prolonged solvent evaporation time is truly effective by providing sufficient 
time for the interdiffusion between the donor and acceptor phases.

The diffusion behavior of PCBM into P3HT crystalline domains is also investigated by changing the regioregularity of the P3HT material. For this purpose, we have prepared devices composed of higher regioregularity (rr)-P3HT, $>98 \%$, instead of $91 \%$ rr-P3HT. Figure 3c shows that the device performances are significantly decreased after changing the rr of P3HT. We use Figure $3 \mathrm{~d}$ to explain this result. 98\% rr-P3HT may provide better crystallinity of P3HT, but it will also leave smaller amorphous region for PCBM molecules to migrate and diffuse, thereby leading to insufficient acceptor domains. Our results also support the recent findings from other research group that PCBM diffuses through the amorphous region of P3HT during annealing process. ${ }^{[14,17]}$

The advanced heterojunction structure, enabled by the ESSESS process in the thick PV cell, is further analyzed by determining the carrier mobility using photo-CELIV method. The photo-CELIV is an efficient method permitting us to estimate the carrier mobility and recombination behavior in low conductivity materials at the same time. ${ }^{[18,19]}$ The detailed information about this method is explained in Experimental section. We note there are certain considerations for using CELIV to determine the carrier mobility; but it has been widely used due to a number of advantages over other methods. ${ }^{[20,21]}$ As shown in Figure 3f, the device fabricated by two steps consecutive ESSENCIAL processes (ESS-ESS) shows the highest mobility, about 5 times higher than the thermally annealed BHJ device. Even Spin-ESS device shows about two times higher mobility than the thermally annealed BHJ device.

Based on the photo-CELIV transient signals, the bimolecular recombination behavior of photo-generated carriers is studied. The recombination loss in organic PV cells is one of the most critical factors limiting the photo-generated current and fill factor of the devices. ${ }^{[22,23]}$ It also has effect on the open circuit voltage due to its nonradiative mechanism in organic PV cells. ${ }^{[24]}$ The recombination losses are classified into three categories. ${ }^{[10]}$ The exciton recombination, which happens when exciton are recombined before reaching donor-acceptor interface, is usually negligible in efficient BHJ system, because domain size is on the order of exciton diffusion length. The charge transfer (CT) state recombination, which happens when the excitons are geminately recombined from the CT state at the interface, is noticeable in organic PV cells that have low carrier mobility and strong Coulomb interaction. These monomolecular losses are dominant between the short circuit condition and maximum power point. ${ }^{[22]}$ The mobile charge carrier recombination, one means of bimolecular recombination, which happens when the dissociated charges are recombined during their transport, plays a major role around open circuit condition, therefore strongly impact the FF. ${ }^{[22]}$ It is still under debate which mechanism is the most dominant in organic PV cells; however the nanomorphology of donor and acceptor for a given material pair is one of the crucial factors in determining the recombination processes. ${ }^{[25,26]}$ Here, we study the bimolecular type recombination in the device structures fabricated by different processes. This bimolecular recombination rate has following equation (Equation 1). ${ }^{[19]}$ $\frac{d n}{d t}=\frac{d p}{d t}=-\beta(t) n p$

where $n$ and $p$ are the concentration of electron and holes, respectively, and $\beta$ is the bimolecular recombination coefficient. By integrating the photo-CELIV transients, the concentration of extracted charge carriers can be calculated at specific delay time between the excitation light pulse and charge extraction. ${ }^{[18]}$ Figure $4 \mathrm{a}$ shows that the number of photo-generated charge in the ESS-ESS devices is the highest, and Spin-ESS devices also show higher amount of photo-charges than thermally annealed BHJ devices. Furthermore, the bimolecular recombination coefficients of ESS-ESS devices, which are calculated by Equation 1 based on the charge density variation according to the time (Figure $4 \mathrm{~b}$ ), show the lowest value, which means that the bimolecular recombination is significantly suppressed in ESS-ESS devices compared with conventional BHJ structures. We believe this behavior is largely due to the improved heterojunction structure between the donor and acceptor, which facilitates the charge transport while minimizes the charge recombination.

In high performance BHJ systems such as P3HT:PCBM blend, a non-Langevin type bimolecular recombination, which has much longer carrier lifetime than the mobility-dependent Langevin type bimolecular recombination $\left(\beta_{\mathrm{L}}\right)$, has been repo rted. ${ }^{[22,23,26-28]}$ In low mobility materials such as organic semiconductors including conjugated polymers, Langevin type recombination is usually observed, ${ }^{[29]}$ and this is one of the critical factors limiting the fill factor of hundreds of nanometers thick BHJ PV cells. Because our devices systems are in a few hundreds of nanometers thick range, the calculated recombination coefficients are compared with their Langevin type recombination coefficients; $\beta_{\mathrm{L}}=e\left(\mu_{\mathrm{n}}+\mu_{\mathrm{p}}\right) / \varepsilon \varepsilon_{\mathrm{o}}$, where $e$ is elementary charge, $\varepsilon \varepsilon_{\mathrm{o}}$ is permittivity of material, $\mu_{\mathrm{n}}$ and $\mu_{\mathrm{p}}$ are electron and hole mobility. ${ }^{[30]}$ The estimated ratios of $\beta$ to $\beta_{\mathrm{L}}$ are $1 \times 10^{-1}, 4 \times 10^{-2}$, and $7 \times 10^{-3}$ for thermal BHJ, Spin-ESS, and ESS-ESS, respectively. The ratio in our $320 \mathrm{~nm}$ thick $\mathrm{BHJ}$ devices $\left(1 \times 10^{-1}\right)$ is higher than those reported in the literatures $\left(10^{-2} \sim 10^{-4}\right)^{[23,28,31]}$ based on $\sim 100 \mathrm{~nm} \mathrm{BHJ} \mathrm{thick} \mathrm{structures,} \mathrm{which} \mathrm{is} \mathrm{another} \mathrm{indi-}$ cation of the more random charge motion due to the non-ideal D/A morphology in the thick layer. Remarkably the ratio of ESSESS made hundreds of nm thick device is the smallest and compatible to the literature reported for the much thinner devices. This means that the Langevin type bimolecular recombination is efficiently suppressed in our thick ESS-ESS device. This effect can again be explained by considering the bi-continuous charge highway system, which minimizes the random charge motion that is responsible for the Langevin type recombination.

Finally the optimized heterojunction morphology in ESSESS devices is verified by calculating internal quantum efficiency (IQE). Recently, Mcgehee et al. reported a method to precisely calculate the IQE by considering parasitic absorption from the reflection spectrum of the PV cell devices. ${ }^{[32]}$ The parasitic absorption in polymer PV cell structure was calculated by transfer matrix method and it was eliminated from the experimentally measured reflection spectrum of PV cell. Figure $4 \mathrm{c}$ shows the calculated absorption in photoactive layer, and the measured external quantum efficiency (EQE) is shown in inset of Figure 4d. It is interesting to note the relatively flat EQE for 

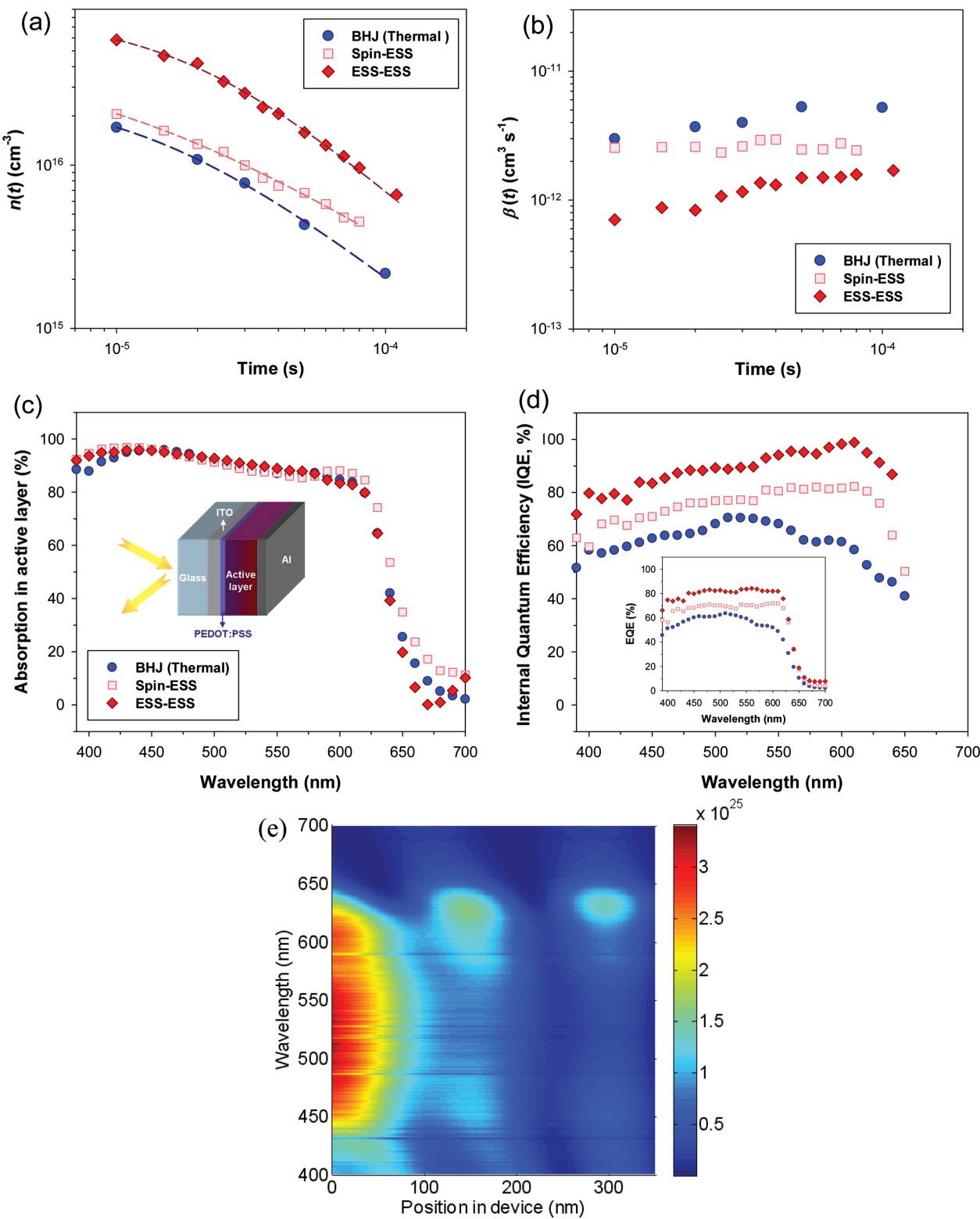

Figure 4. (a) The concentration of extracted charge carrier versus delay time between light pulse and extraction according to the different processing methods. (b) The calculated bimolecular recombination coefficient versus delay time according to the different processing methods. (c) The absorption efficiency in photoactive layer. The parasitic absorption effect calculated by transfer matrix method was eliminated from the reflection spectrum of the real device. (d) Calculated internal quantum efficiency (IQE). Inset of (d) is external quantum efficiency (EQE) measured by IPCE. (e) The calculated photon absorption according to the thickness of film under AM 1.5 sun spectrum. The position having 0 value is ITO anode-side.

the ESS-ESS device as compared with BHJ device, especially significantly increased $\mathrm{EQE}$ at longer wavelength range $(\sim 625 \mathrm{~nm}$, close to the absorption edge of P3HT material). This could be related to the reduced bandgap due to the polymer chain aggregation in the P3HT fibrils, which correlates very well to the lower $V_{\mathrm{oc}}$ of the ESS-ESS devices. Integrating the EQE curves give us photo-currents, which are within $5 \%$ deviation of the measured $J_{\mathrm{sc}}$. Following the relation $(\mathrm{EQE}=$ Absorption in active layer $\times$ IQE), IQE signals are calculated in Figure 4d. As shown, ESS-ESS device having optimized morphology shows the highest IQE value. Importantly, the IQE value even approaches $100 \%$ around $600 \mathrm{~nm}$ wavelength, which means the most of the photo-generated excitons are fully dissociated and charges transported to the respective electrodes without much recombination. Figure 4e shows the calculated photon absorption according to the film thickness. As expected, the overall photon absorption is maximized at the anode interface to which sunlight is exposed, and the spectrum is relatively flat between 450 and $630 \mathrm{~nm}$ wavelength. However, the absorption around the middle of the device ( $\sim 170 \mathrm{~nm}$ thickness), in which it is expected that the recombination of charges is minimized due to their shorter travel distances to each electrode than at the 
anode interface, ${ }^{[33]}$ shows relatively high values around $600 \mathrm{~nm}$ wavelength. This may be a potential reason to the highest IQE at $600 \mathrm{~nm}$, and this is still under investigation.

In summary, we developed a new bilayer-like approach to produce the efficient photoactive heterojunction nanostructure by maximizing the interdiffusion of donor and acceptor materials. The optimized morphology has improved crystallinity, leading to significantly improved carrier transportation, and lower recombination coefficient, resulting in high IQE approaching almost $100 \%$ at certain spectral range. Consequently, the devices based on this type of structure show significantly high photocurrent (about 50\% higher than that in thermally annealed BHJ devices) and fill factor. We expect that this new type of structure introduced in this work can help us further understand the charge transport properties in the D/A heterostructures and improve the performance of polymer PV cells.

\section{Experimental Section}

Polymer PV cells fabrication: Polymer PV cells have the following configuration: transparent substrate/ITO/PEDOT:PSS/P3HT:PCBM/ $\mathrm{LiF} / \mathrm{Al}$. The thermally annealed devices were fabricated by spin-casting the blend solution (dissolved in dichlorobenzene) onto a PEDOT:PSScoated ITO substrate and subsequently annealing at $130^{\circ} \mathrm{C}$ for $20 \mathrm{~min}$. PV cells with advanced heterojunction structure were fabricated by two consecutive ESSENCIAL processes. The first electron-donor phase was formed by pressing the $\mathrm{P} 3 \mathrm{HT}$ solution (dissolved in chlorobenzene) onto the PEDOT:PSS-coated ITO substrate using a modified PDMS silicone film, which is gas-permeable and solvent-resistant with minimal deformation. ${ }^{[34]}$ The thickness of the film fabricated by the ESSENCIAL method was controlled by changing the concentration of the solution and the applied pressure, and the solvent evaporation time could be changed from tens of minutes to a few seconds depending on the applied pressure. All the film thicknesses were measured by Dektak Surface Profilometer. The solvent evaporation time of the first ESSENCIAL process to form electron-donor phase did not affect the morphology and final device performances. Thermal annealing treatment was not needed to the $\mathrm{P} 3 \mathrm{HT}$ layer formation. On top of $\mathrm{P} 3 \mathrm{HT}$ electron-donor phase, the electron-acceptor PCBM phase was formed by the second ESSENCIAL process using dichloromethane as a semi-orthogonal solvent. The solvent evaporation time of the second ESSENCIAL process was over $10 \mathrm{~min}$. The migration of PCBM molecules into $\mathrm{P} 3 \mathrm{HT}$ phase and the resultant intermixing were maximized during this process without any thermal annealing treatment. LiF $(1 \mathrm{~nm})$ and Al cathode $(100 \mathrm{~nm})$ deposition through circular shape mask with $1 \mathrm{~mm}$ diameter on the hetrojunction structure completed the device fabrication. Over 50 devices were prepared for each process condition.

The device performances were measured with Keithley 2400 system by illuminating the polymer PV cells using Oriel solar simulator (equipped with Xenon lamp and AM $1.5 \mathrm{G}$ filter) at the irradiation intensity of $100 \mathrm{~mW} \mathrm{~cm}{ }^{-2}$, calibrated by NREL-certified Si reference cell. The spectral mismatch of the simulated light source was corrected. ${ }^{[35]}$ The differences between the short-circuit current densities obtained by J-V curves and those calculated by EQE signal are within 5\%. Incident-photon-tocollected-charge-carrier efficiency (IPCE) spectra for EQE were measured with a $150 \mathrm{~W}$ Oriel solar simulator light source, an Acton Research Corp. Spectra-Pro 275 monochromator, a chopper wheel and a Stanford Research 830 lock-in amplifier. The photocurrents were measured and normalized against a Hammamatsu Si photodiode reference, and long pass filters were used to block transmission of any stray secondary or tertiary diffractions outside of the range of interest.

Photo-CELIV measurement: A short $\mathrm{N}_{2}$ gas pulsed laser $(337 \mathrm{~nm}$, PTI GL-3300) with a $1.5 \mathrm{~ns}$ pulse width and a $2 \mathrm{~J} \mathrm{~m}^{-2}$ pulse energy was illuminated on device samples to generate charge carriers within the photoactive layers. The generated charge carriers were then extracted using a linearly increasing reverse bias pulse (sawtooth-shaped voltage pulse). The voltage pulse was created using an HP 3314A function generator. The delay time ( $\left.t_{\text {del }}\right)$ between the laser flash and the triangular bias pulse was in the range of 1 to $200 \mu \mathrm{s}$, which was controlled by an SRS DG535 digital delay/pulse generator (Stanford Research Systems). An optical sample stage and two micro manipulators were used to establish electrical contact with the anode/cathode through which the voltage pulses (widths of $t_{\mathrm{p}}=100 \mu \mathrm{s}$ with a $10 \mathrm{kHz}$ frequency) were applied. The peak voltage in the reverse direction was approximately $V_{a}=$ $1 \mathrm{~V}-2 \mathrm{~V}$ and was applied to the Al cathode electrode with a varied offset bias $V_{\text {off }}$ in the forward direction. The offset bias was set to compensate for the intrinsic built-in potential. The resulting current was monitored as a function of time using the $50 \Omega$ input of a digital storage oscilloscope (DSO7054A, Agilent Technologies). All photo-CELIV measurements were performed at room temperature.

When a reverse, sawtooth-shaped bias is applied with an increasing rate $A\left(\mathrm{~V} \mathrm{~s}^{-1}\right)$ in the dark to low conductivity materials such as polymer PV cells, a rectangular-shaped current transient having a constant value is measured as an electrical signal. This constant current value is a capacitive displacement current $j(0)\left(\mathrm{C} \mathrm{s}^{-1}\right)$. In this condition, when the device is exposed to pulsed laser excitation, photo-charges are generated in the photoactive layer, and they either recombine or are extracted by the electric field. By measuring $t_{\max }$, which occurs at the maximum photocurrent, and comparing the ratio of extracted current $(\Delta j)$ to constant current $(j(0))$, we can calculate the mobility of carriers using Equation 2 [18].

$\mu=\frac{2 d^{2}}{3 A t_{\max }^{2}\left(1+0.36 \frac{\Delta j}{j(0)}\right)}(\Delta j \leq j(0))$

where $d$ is the thickness of photoactive layer.

Morphology Characterization: Glazing incidence wide angle X-ray scattering (GIWAXS) was performed on Beamline X9 at Brookhaven National Laboratory. The experimental conditions of X-ray were $13.5 \mathrm{keV}$ and $0.918 \AA$. The samples were measured at $0.3^{\circ}$ over the critical angle to analyze the organization of polymer chains in the bulk. Dynamic secondary ion mass spectrometry (DSIMS) signals were measured in Materials Research Laboratory at University of California, Santa Barbara. The samples were measured using $2 \mathrm{kV} \mathrm{O} 2+$ primary ions, detecting negative secondary ions from the $15 \%$ of the center of the crater area. The crater had a rectangle, $200 \mu \mathrm{m} \times 230 \mu \mathrm{m}$ in size. $200 \mathrm{eV}$ electron beam was used for charge neutralization. All the signals in first $400 \mathrm{~s}$ sputtering time were from polystyrene film, which was deposited on the samples by floating method not to destroy the film morphology.

\section{Supporting Information}

Supporting Information is available from the Wiley Online Library or from the author.

\section{Acknowledgements}

This work was supported as part of Center for Solar and Thermal Energy Conversion, an Energy Frontier Research Center funded by the U.S. Department of Energy, Office of Science, Basic Energy Sciences under Award DE-SC0000957. H. J. P. acknowledges a Rackham Predoctoral fellowship from the University of Michigan. The authors would like to thank Dr. Lin Yang at Brookhaven National Laboratory for help with GIWAXS measurements and Dr. Tom Mates at University of California, Santa Barbara for help with DSIMS measurements.

Received: March 4, 2013

Revised: March 20, 2013

Published online: May 23, 2013 
[1] P. Peumans, A. Yakimov, S. R. Forrest, J. Appl. Phys. 2003, 93, 3693.

[2] H. Ma, H.-L. Yip, F. Huang, A. K.-Y. Jen, Adv. Funct. Mater. 2010, 20, 1371

[3] Z. He, C. Zhong, X. Huang, W.-Y. Wong, H. Wu, L. Chen, S. Su, Y. Cao, Adv. Mater. 2011, 23, 4636.

[4] W.-Y. Wong, X.-Z. Wang, Z. He, A. B. Djuri, C.-T. Yip, K.-Y. Cheung, H. Wang, C. S. K. Mak, W.-K. Chan, Nat. Mater. 2007, 6, 521.

[5] G. Li, V. Shrotriya, J. Huang, Y. Yao, T. Moriarty, K. Emery, Y. Yang, Nat. Mater. 2005, 4, 864.

[6] M.-S. Kim, B.-G. Kim, J. Kim, ACS Appl. Mater. Inter. 2009, 1, 1264.

[7] H. J. Park, M.-G. Kang, S. H. Ahn, L. J. Guo, Adv. Mater. 2010, 22, E247.

[8] B. Walker, A. B. Tamayo, X.-D. Dang, P. Zalar, J.-H. Seo, A. Garcia, M. Tantiwiwat, T.-Q. Nguyen, Adv. Funct. Mater. 2009, 19, 3063.

[9] L. Blankenburg, K. Schultheis, H. Schache, S. Sensfuss, M. Schrödner, Sol. Energy Mater. Sol. Cells 2009, 93, 476.

[10] A. Pivrikas, H. Neugebauer, N. S. Sariciftci, IEEE J. Sel. Top. Quantum Electron. 2010, 16, 1746.

[11] B.-G. Kim, E. J. Jeong, H. J. Park, D. Bilby, L. J. Guo, J. Kim, ACS Appl. Mater. Inter. 2011, 3, 674.

[12] M.-G. Kang, H. J. Park, S. H. Ahn, T. Xu, L. J. Guo, IEEE J. Sel. Top. Quantum Electron. 2010, 16, 1807.

[13] K. H. Lee, P. E. Schwenn, A. R. G. Smith, H. Cavaye, P. E. Shaw, M. James, K. B. Krueger, I. R. Gentle, P. Meredith, P. L. Burn, Adv. Mater. 2011, 23, 766.

[14] D. Chen, F. Liu, C. Wang, A. Nakahara, T. P. Russell, Nano Lett. 2011, 11, 2071.

[15] S.-S. Kim, S.-I. Na, J. Jo, G. Tae, D.-Y. Kim, Adv. Mater. 2007, 19, 4410.

[16] W. Chen, M. P. Nikiforov, S. B. Darling, Energy Environ. Sci. 2012, 5, 8045 .
www.MaterialsViews.com

[17] N. D. Treat, M. A. Brady, G. Smith, M. F. Toney, E. J. Kramer, C. J. Hawker, M. L. Chabinyc, Adv. Energy Mater. 2011, 1, 82.

[18] G. Juška, K. Arlauskas, M. Viliunas, Phys. Rev. Lett. 2000, 84, 4946.

[19] A. J. Mozer, G. Dennler, N. S. Sariciftci, M. Westerling, A. Pivrikas, R. Österbacka, G. Juška, Phys. Rev. B 2005, 72, 035217.

[20] H. J. Park, H. Kim, J. Y. Lee, L. J. Guo, Energy Environ. Sci., DOI: 10.1039/C3EE24410E.

[21] A. Baumann, J. Lorrmann, D. Rauh, C. Deibel, V. Dyakonov, Adv. Mater. 2012, 24, 4381.

[22] S. R. Cowan, A. Roy, A. J. Heeger, Phys. Rev. B 2010, 82, 245207.

[23] A. Pivrikas, N. S. Sariciftci, G. Juška, R. Österbacka, Prog. Photovolt: Res. Appl. 2007, 15, 677.

[24] K. Vandewal, K. Tvingstedt, A. Gadisa, O. Inganäs, J. V. Manca, Nat. Mater. 2009, 8, 904.

[25] R. A. Street, S. Cowan, A. J. Heeger, Phys. Rev. B 2010, 82, 121301 (R).

[26] L. Liu, G. Li, Solar Energy Mater. Solar Cells 2011, 95, 2557.

[27] L. J. A. Koster, V. D. Mihailetchi, P. W. M. Blom, Appl. Phys. Lett. 2006, 88, 052104.

[28] C. G. Shuttle, B. O. Regan, A. M. Ballantyne, J. Nelson, D. D. C. Bradley, J. R. Durrant, Phys. Rev. B 2008, 78, 113201.

[29] N. Karl, G. Sommer, Phys. Status Solidi A 1971, 6, 231.

[30] P. Langevin, Ann. Chim. Phys. 1903, 28, 433.

[31] C. Deibel, A. Baumann, V. Dyakonov, Appl. Phys. Lett. 2008, 93, 163303.

[32] G. F. Burkhard, E. T. Hoke, M. D. McGehee, Adv. Mater. 2010, 22, 3293.

[33] J. D. Kotlarski, P. W. M. Blom, Appl. Phys. Lett. 2012, 100, 013306.

[34] C. Pina-Hernandez, J.-S. Kim, L. J. Guo, P.-F. Fu, Adv. Mater. 2007, 19, 1222.

[35] V. Shrotriya, G. Li, Y. Yao, T. Morarty, K. Emery, Y. Yang, Adv. Funct. Mater. 2006, 16, 2016 